\title{
A STRATEGY FOR VEHICLE CONTROL AUGMENTATION VIA CONTROL BANDWIDTH PHASE SHAPING APPROACH
}

\author{
Atsushi Satoh* Kenji Sugimoto* \\ * Graduate School of Information Science, Nara Institute \\ of Science and Technology, 8916-5 Takayama, Ikoma, \\ 630-0192 JAPAN. \{s-atushi, kenji\}@is.naist.jp
}

\begin{abstract}
This paper discusses a vehicle control augmentation strategy bringing into focus the phase property, and proposes a control bandwidth phase shaping technique which is applicable to control augmentation system design. This paper points out that the problem setting of the control augmentation system design and structure/control integrated design has similarity. As expected from the similarity, it is observed that the phase crossover bandwidth of vehicle dynamics has crucial effect to the tracking performance for pilot control input. Furthermore, a finite bandwidth phase shaping technique via dissipative control theory is proposed. Namely, the phase shaping in control bandwidth is achieved with no phase constraint in the higher frequency range. Therefore, a control augmentation system which achieves the phase shaping in the control bandwidth is easily designed by the presented technique. Copyright ${ }^{\circledR} 2005$ IFAC
\end{abstract}

Keywords: Phase Crossover Bandwidth, Finite Bandwidth Loop Shaping, Integrated Design, Dissipative Control, Control Augmentation System.

\section{INTRODUCTION}

Manned vehicles, such as cars or aircrafts, are virtually considered as the feedback system which consists of the vehicle and the pilot. The vehicles are desired to have some appropriate open-loop properties for easy and precision control by the pilots. What is called "handling quality" mainly represents the control performance of the closed system which consists of the vehicle and the pilot, and several criteria of vehicle dynamical properties for good handling quality are proposed via experimental investigations (Bihrle Jr., 1966; Neal and Smith, 1971; Military Specification, 1990).

However, improving the handling quality by vehicle structural design alone is often difficult, because costs or other practical restrictions strongly constrain the structure. Therefore, additional con- trol systems aiming at improvement of handling quality are widely implemented nowadays. Examples are power steering, traction control system, pitch/yaw damper, and more advanced feedback control systems. Let us generally call these systems CAS (control augmentation system).

To evaluate the handling quality for a given vehicle and CAS without human pilot, a practical idea of "optimal control model" was proposed (Kleinman et al., 1970; Davidson and Schmidt, 1992). This is a pilot model based on optimal control theory, and the handling quality is estimated from the achieved optimal control performance.

Although this idea is successfully applied to handling quality analysis (Schmidt, 1979; Doman and Anderson, 2000), it is not as effective for CAS design. Because, the optimal control model ob- 


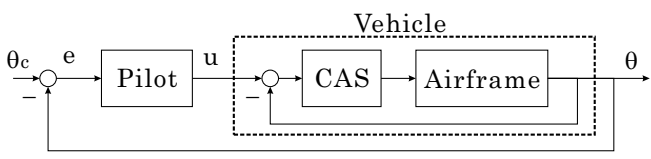

Fig. 1. Aircraft pitch tracking control.

viously depends on not only the vehicle but the CAS, which we want to design from now on.

This means that CAS should not be designed to improve the control performance for a fixed pilot model, but the best performance for every pilot model. As is shown in the later, this problem setting is similar to that of the structure/control integrated design, which is well-known in the area of mechanical control system design (Onoda and Haftka, 1987; Grigoriadis and Wu, 1997; Iwasaki, 1999). This similarity suggests that the CAS design is a class of BMI problem and difficult to obtain a globally optimal solution, even if the pilot is simply modeled as an optimal controller.

However, Iwasaki (1999) proposed a tractable integrated design strategy by separation. The point of Iwasaki's strategy is that the achievable closed-loop performance definitely depends on a certain open-loop property, therefore we can achieve near-optimal integrated design without solving BMI problem. From numerical analysis and theoretical results, Iwasaki concluded that the phase crossover bandwidth of the plant is crucial for optimal closed-loop $H_{2}$ tracking performance. Therefore, from the similarity of both problem settings, it is natural to expect that the phase crossover bandwidth of a vehicle dynamics is the essential property for the CAS design.

This paper discusses a vehicle control augmentation strategy bringing into the focus the phase crossover bandwidth, and proposes a new CAS design via the control bandwidth phase shaping technique. In the first half of this paper, the relationship of the phase crossover bandwidth of the vehicle dynamics and the achievable optimal handling quality is considered by $H_{2}$ performance analysis, however this approach was proposed in the authors' preliminary work (Satoh et al., 2004).

Then, in the latter half, a finite bandwidth phase shaping technique via dissipative control theory is proposed. Namely, gain and phase shaping in the control bandwidth is achieved with no phase constraint in the higher frequency range. Therefore, a control augmentation system which achieves the phase shaping in the control bandwidth is easily designed by the proposed technique.

Let us define the crossover frequency which is often used in this work. For a SISO transfer function $H(s)$, the smallest $\omega_{p}>0$ such that $\angle H\left(j \omega_{p}\right)=$ $-\pi$ is called Phase crossover frequency.

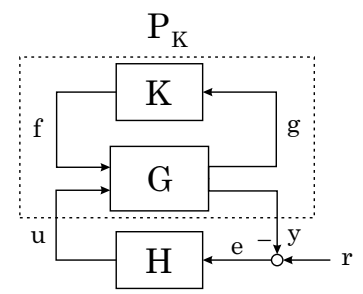

Fig. 2. CAS design problem.

\section{CONTROL AUGMENTATION SYSTEM DESIGN}

A typical example of CAS design is an aircraft pitch tracking control (Fig. 1). The CAS represents the dynamics of the attached controller. The airframe represents the dynamics of the vehicle except for the CAS. Let the airframe is given and we can only design the CAS to change the total dynamical property of the vehicle. Then, the CAS is virtually considered as the design parameter of the vehicle. Furthermore, the pilot is virtually considered as the feedback controller to the vehicle.

Following the idea of optimal control model, let us consider to model an well-motivated and experienced (or "optimized") pilot as a simple $\mathrm{H}_{2}$ optimal controller which achieves servo tracking control of $\theta$ (pitch angle) to $\theta_{c}$ (pitch angle command) in this work.

\section{CAS DESIGN FROM THE VIEWPOINT OF INTEGRATED DESIGN}

Then, let us consider the CAS design for servo tracking control from the viewpoint of the integrated design. For a given $G$, consider the feedback system in Fig. 2. Assume that $K, G$ and $H$ are finite dimensional LTI systems. The $P_{K}$ denotes the extended plant, where $u, y$ are control input/output and $r, e$ are reference input/error.

Note that $K, G$, and $H$ correspond to a CAS, an airframe and a pilot model respectively. Therefore, the design objective is to find the subsystems $K$ and $H$ such that optimize the optimal $H_{2}$ tacking performance from $r$ to $e$, for the given $G$.

By using the above framework, let us define the CAS design problem as follows (Satoh et al., 2004).

Problem 1. (CAS design problem). Let $G$ and $\gamma_{u}>0$ be given. Find $H, K$ which minimize the optimal $\mathrm{H}_{2}$ tracking performance;

$$
\gamma_{e}\left(\gamma_{u}\right):=\min \left\|T_{e}\right\|_{2} \text { such that }\left\|T_{u}\right\|_{2}<\gamma_{u},
$$

where $\left[\begin{array}{l}e \\ u\end{array}\right]=\left[\begin{array}{l}T_{e} \\ T_{u}\end{array}\right] r$.

The index $\gamma_{u}$ corresponds the limit of pilot control power (e.g. average power of the control stick 


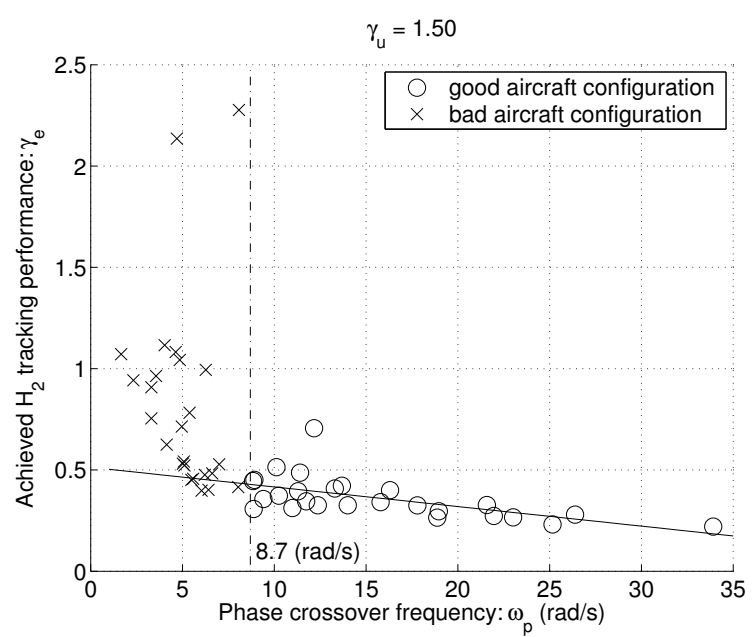

Fig. 3. Adequate phase crossover bandwidth for optimal $\mathrm{H}_{2}$ performance.

force). If Problem 1 is feasible for the given $\gamma_{u}$, the $H$ is the optimal pilot model in the meaning of $\mathrm{H}_{2}$ performance, the $K$ is an optimal CAS and the $\gamma_{e}$ represents the achieved handling quality of the vehicle (with CAS).

If the $K$ is restricted to a passive component (i.e. $K:=$ block-diag $\left[p_{1} I, \cdots, p_{r} I\right]$ with design parameters $\left.\left(p_{1}, \ldots, p_{r}\right)\right)$, Problem 1 is reduced to an integrated design problem of structure and control system. This suggests that Problem 1 is a class of BMI problem and difficult to design an optimal CAS with a reasonable computing cost in general, even if the systems are LTI and the pilot is simply modeled as an $H_{2}$ controller.

However, Iwasaki (1999) proposed a tractable design strategy for the integrated design version of Problem 1, by separation. The separation strategy means that first $K$ is designed to make $P_{K}$ meet the open-loop property which is desirable to achieve optimal closed-loop performance, then $H$ is designed to achieve optimal closed-loop performance. Iwasaki considered the $H_{2}$ tracking performance for the step reference input $r$, and concluded that the phase crossover frequency of $P_{K}$ is desired to be higher than control bandwidth.

\section{BANDWIDTH ANALYSIS AND THE OPTIMAL CLOSED-LOOP PERFORMANCE}

From the similarity between the problem setting of CAS design and integrated design, it is natural to expect that the phase crossover bandwidth of $P_{K}$ is essential for the CAS design as well.

Then, let us reconsider the Neal and Smith's (1971) handling quality investigation from the integrated design point of view. For an airframe and 52 variations of the CAS from their work, the authors solved the $\mathrm{H}_{2}$ optimization problem (1) for the pitch tracking control (Fig. 1), and

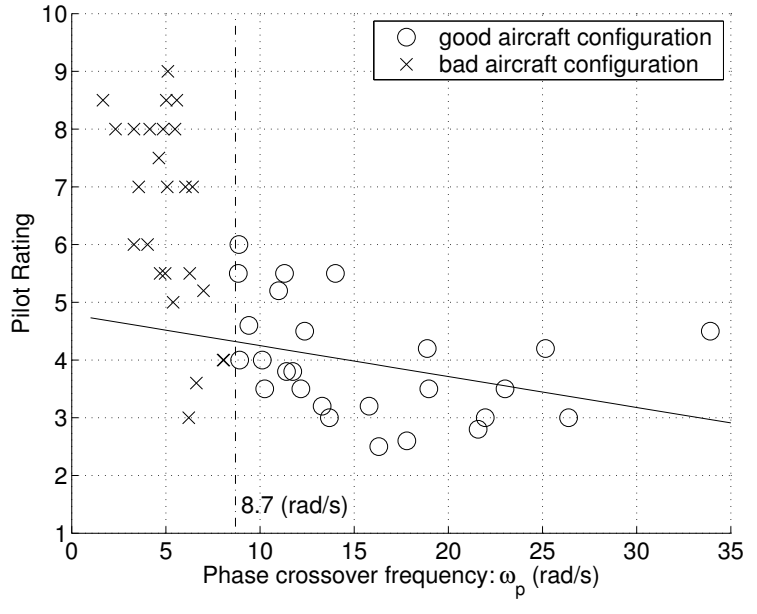

Fig. 4. Pilot Rating of each configuration.

evaluated the handling quality by the achieved $\mathrm{H}_{2}$ performance $\gamma_{e}$. In Fig. 3, the plotted circles or crosses correspond to each configuration (i.e. 52 combinations of the airframe and the CAS). We can observe that $H_{2}$ performance is optimized for the configurations which has about $\omega_{p} \geq 8.7$ $(\mathrm{rad} / \mathrm{s})$ (plotted as the circles). In the above meaning, let us call them "good" configurations.

On the other hand, Neal and Smith (1971) evaluated the handling quality by "Pilot Rating" (PR). The rating is a numerical index which is systematically determined from pilot comments, and primary depends on how quickly and precisely the pilot could control pitch angle. The range of PR is from 1 (best) to 10 (worst). In Fig. 4, the phase crossover frequency $\omega_{p}$ and PR for each aircraft configuration are plotted. We can observe that PR is also optimized for "good" configurations, which is characterized by the phase crossover bandwidth. The average PR for "good" configurations is 3.95.

From the above bandwidth analysis, we can observe that

(i) If the phase crossover bandwidth of the vehicle is sufficiently large, then both $H_{2}$ tracking performance and PR are optimized.

(ii) For this vehicle, $\omega_{p} \simeq 8.7(\mathrm{rad} / \mathrm{s})$ is enough for optimal $\mathrm{H}_{2}$ tracking performance and PR.

These observations suggests that the separation strategy is effective for the CAS design. From the observation (2), it seems that the desired control bandwidth for optimal PR is about $8.7(\mathrm{rad} / \mathrm{s})$ for this vehicle. This gives us the CAS design specification from integrated design point of view.

\section{FINITE BANDWIDTH PHASE SHAPING AND APPLICATION TO CAS DESIGN}

The above discussion suggests that the phase crossover bandwidth of the vehicle is crucial 


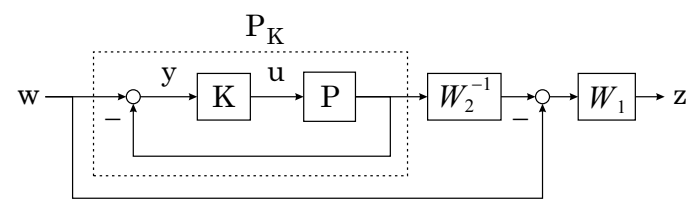

Fig. 5. Finite bandwidth phase shaping problem.

for handling quality augmentation in servo control like tasks. It is pointed out that the phase crossover bandwidth of the plant almost coincides with the achievable servo bandwidth in the integrated design (Iwasaki, 1999). This also suggests the plant phase property in the very high frequency is noncrucial for performance. Therefore, phase shaping in the finite frequency range is important for the design strategy by separation.

Iwasaki et al. (2000) proposed the finite frequency KYP lemma and applied to the control/structure integrated design. This approach does not need a weighting function for the phase shaping. However, the feedback design is reduced to BMI problem in general. Sakamoto and Suzuki (1996) proposed the $\gamma$-positive realness which introduce the measure of positive realness, $\gamma$. This idea was successfully applied to CAS design by the authors (Satoh et al., 2004). The feedback design is tractable (i.e. LMI), however, it results in excessive phase shaping in all frequency range.

Therefore, another tractable phase shaping technique in the control bandwidth is proposed in this work via dissipative control theory.

Consider proper SISO transfer functions $Q, W$, and assume that $W(Q-1) \in \mathbf{R H}_{\infty}$ and $W$ does not have zero on the imaginary axis. The equivalence of the next two statements follows from the definition of $H_{\infty}$ norm.

(i) The next $H_{\infty}$ norm condition holds.

$$
\|W(s)(Q(s)-1)\|_{\infty}<1 .
$$

(ii) For all $\omega \in \mathbf{R}$.

$$
\left[Q(j \omega)^{*} 1\right]\left[\begin{array}{cc}
-1 & 1 \\
1 & |W(j \omega)|^{-2}-1
\end{array}\right]\left[\begin{array}{c}
Q(j \omega) \\
1
\end{array}\right]>0 .
$$

The inequality (3) means that the Nyquist plot of $Q$ always resides in the disk whose center is $1+0 j$ and radius is $1 /|W(j \omega)|$. Note that the radius depends on $\omega$.

Theorem 1. If (2) holds, then ${ }^{\forall} \omega \in \Omega_{c}$,

$$
\begin{array}{r}
|\angle Q(j \omega)|<\arcsin \left(|W(j \omega)|^{-1}\right) . \\
1-\frac{1}{|W(j \omega)|}<|Q(j \omega)|<1+\frac{1}{|W(j \omega)|},
\end{array}
$$

Furthermore, ${ }^{\forall} \omega \in \bar{\Omega}_{c}$,

$$
|Q(j \omega)|<1+\frac{1}{|W(j \omega)|},
$$

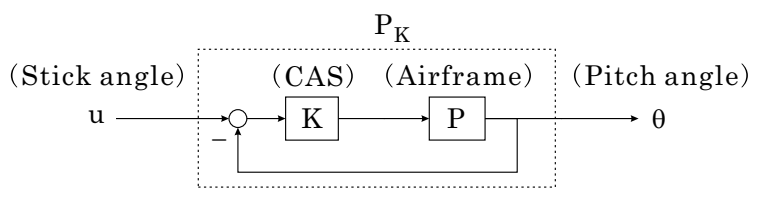

Fig. 6. CAS design.

where

$$
\Omega_{c}:=\{\omega \in \mathbf{R}:|W(j \omega)| \geq 1\} .
$$

Proof 1. Let us show a brief proof. From the condition (2), for all $\omega \in \mathbf{R}$,

$$
\begin{aligned}
& |Q(j \omega)-1|<|W(j \omega)|^{-1} \\
\Leftrightarrow & \{\Re(Q(j \omega)-1)\}^{2}-|W(j \omega)|^{-2} \\
& <-\{\Im(Q(j \omega)-1)\}^{2} .
\end{aligned}
$$

The right term of (9) is less than or equal to zero. Therfore the next inequalities hold for all $\omega \in \mathbf{R}$.

$$
-\frac{1}{|W(j \omega)|}<\Re(Q(j \omega))-1<\frac{1}{|W(j \omega)|} .
$$

The first two inequalities of (10) implies the first two inequalities of (5) hold for all $\omega \in \Omega_{c}$. On the other hand, from (8),

$$
\begin{aligned}
& |Q(j \omega)|^{2}<\left(1+\frac{1}{|W(j \omega)|}\right)^{2} \\
& +2\left(\Re(Q(j \omega))-1-\frac{1}{|W(j \omega)|}\right) .
\end{aligned}
$$

The second term of the right hand side of (11) is less than zero from (10). This implies (6) and the latter two inequalities of (5) hold. The condition (4) is proved via geometric relation of (3).

Note that no phase condition holds for $\omega \in \bar{\Omega}_{c}$ from theorem 1 . Then, let us dicuss the finite bandwidth phase shaping by feedback control. For a given SISO plant $P$ and weighting functions $W_{1}, W_{2}$, consider the next $H_{\infty}$ problem (Fig. 5).

$$
\begin{array}{r}
\left\|W_{1}\left(W_{2}^{-1} P_{K}-1\right)\right\|_{\infty}<1, \\
P_{K}:=(1+P K)^{-1} P K .
\end{array}
$$

The generalized plant is given as follows.

$$
\left[\begin{array}{l}
z \\
y
\end{array}\right]=\left[\begin{array}{cc}
-W_{1} & W_{1} W_{2}^{-1} P \\
1 & -P
\end{array}\right]\left[\begin{array}{l}
w \\
u
\end{array}\right] .
$$

Note that the weightings should be selected to make $W_{1}$ and $W_{1} W_{2}^{-1} P$ be proper.

The phase shaping bandwidth $\Omega_{c}$ is determined by $W_{1}$, and the phase property in $\Omega_{c}$ is determined by $W_{2}$. If the design problem (12) is solved, then the phase shaping in the specified bandwidth $\Omega_{c}$ is achieved by $K$.

Let us show an illustrative example of the aircraft CAS design (Fig. 6). The plant is from Neal and 

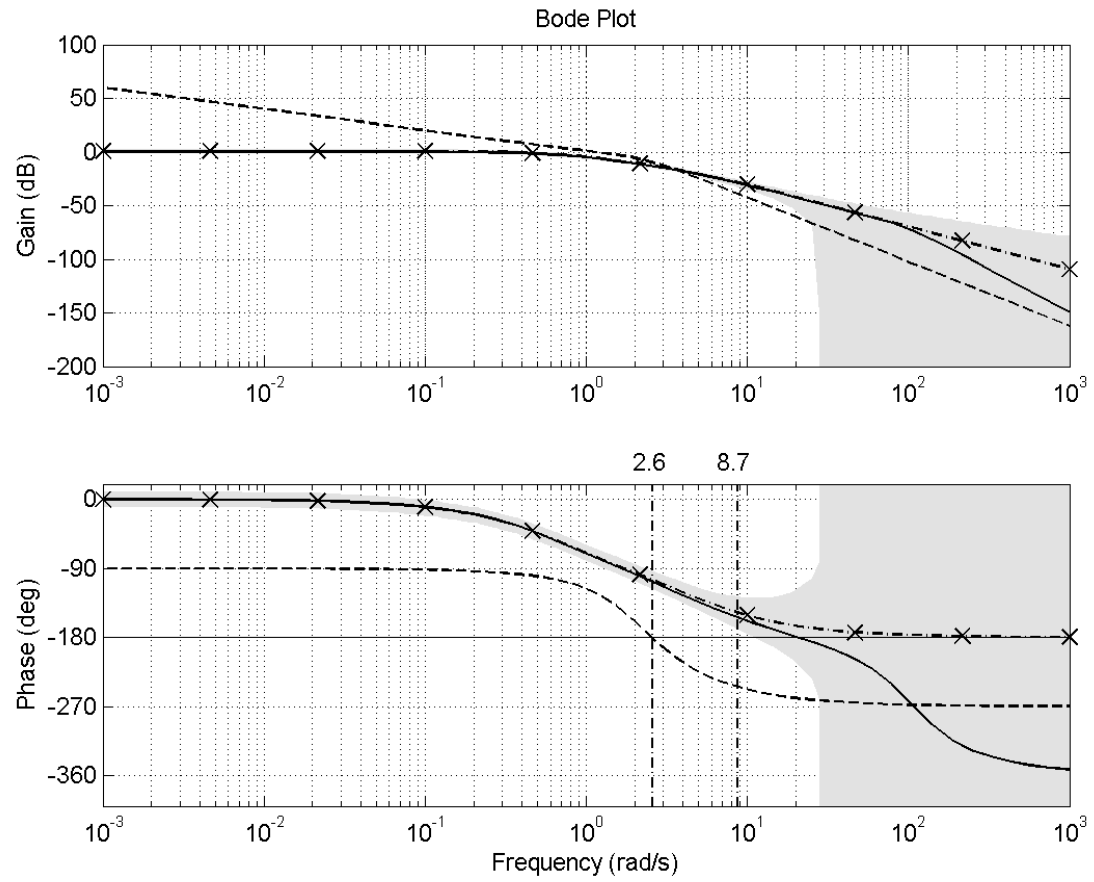

Fig. 7. Design result.

Smith's (1971) investigation (the configuration ' $1 \mathrm{~F}$ '), where elevator actuator is modeled as a first order lag $\left(\tau_{a}=0.5\right)$.

$$
P(s):=\frac{1}{\tau_{a} s+1} \cdot \frac{K_{\theta}\left(\tau_{\theta 2} s+1\right)}{s\left(\frac{1}{\omega_{s p}^{2}} s^{2}+2 \frac{\zeta_{s p}}{\omega_{s p}} s+1\right)} .
$$

Plant parameters are $K_{\theta}=1, \tau_{\theta 2}=1 / 1.25, \omega_{s p}=$ $2.2, \zeta_{s p}=0.69$ respectively.

Following the observation in $\S 4$, let us design the CAS to shape the phase property of the vehicle. The design specification is given as follows.

Specification 1. The phase crossover frequency of the $P_{K}$ is more than $\omega_{p}:=8.7(\mathrm{rad} / \mathrm{s})$.

Considering the specification 1 , the shaping banwidth should be selected as $\Omega_{c}=\{0 \leq \omega \leq \bar{\omega}\}$ for some $\bar{\omega} \geq \omega_{p}$. In this example, $W_{1}$ is selected as

$$
W_{1}(s):=\frac{1 \times 10^{-5} s+28.5}{s+5},
$$

because $\left|W_{1}(j \omega)\right| \geq 1$ for all $\omega$ in

$$
\Omega_{c}=\{0 \leq \omega \leq 28.1(\mathrm{rad} / \mathrm{s})\} .
$$

Then, $W_{2}$ is selected as a second-order lag.

$$
W_{2}(s):=\frac{2}{0.6 s^{2}+3.2 s+1.8} .
$$

This guarantees that the maximum phase lag of $W_{2}$ (18) in $\Omega_{c}$ (17) is less than or equal to 180 (deg.), and $W_{1} W_{2}^{-1} P$ is proper.

If the condition (12) is satisfied, from Theorem 1 , $P_{K}$ satisfies the following phase/gain conditions for all $\omega \in \Omega_{c}(17)$.

$$
\begin{aligned}
& \left|\angle P_{K}(j \omega)-\angle W_{2}(j \omega)\right|<\arcsin \left(\left|W_{1}(j \omega)\right|^{-1}\right), \\
& 1-\frac{1}{\left|W_{1}(j \omega)\right|}<\frac{\left|P_{K}(j \omega)\right|}{\left|W_{2}(j \omega)\right|}<1+\frac{1}{\left|W_{1}(j \omega)\right|} .
\end{aligned}
$$

Furthermore, for all $\omega \notin \Omega_{c}$ (17),

$$
\frac{\left|P_{K}(j \omega)\right|}{\left|W_{2}(j \omega)\right|}<1+\frac{1}{\left|W_{1}(j \omega)\right|} .
$$

From (16) and (18), $\angle W_{2}\left(j \omega_{p}\right)=-147.4$ (deg.) and $\left|\angle P_{K}\left(j \omega_{p}\right)-\angle W_{2}\left(j \omega_{p}\right)\right|<20.9$ (deg.). Since $\angle W_{2}(j \omega)$ is monotonically decreasing function, this guarantees that phase crossover bandwidth of $P_{K}$ is more than $\omega_{p}$.

The $H_{\infty}$ problem (12) is solved by LMI control toolbox in MATLAB R13 SP1. The designed controller is

$$
\begin{aligned}
K(s) & =\left(4230 s^{4}+21320 s^{3}+46150 s^{2}+40930 s\right. \\
& +3.611) /\left(s^{5}+132.8 s^{4}+11670 s^{3}+71820 s^{2}\right. \\
& +68540 s-956.4)
\end{aligned}
$$

We can clearly see the improvement of phase crossover bandwidth in Fig. 7. The broken line is the Bode plot of the $P(15)$. The phase crossover frequency of the $P$ is about $2.6(\mathrm{rad} / \mathrm{s})$, therefore it is not enough for good handling quality. The solid line in Fig. 7 is the Bode plot of the closedloop system $P_{K}$ (13) which is designed via the presented technique, where dash-dot line with marker ' $\mathrm{x}$ ' is the Bode plot of $W_{2}(18)$. We can confirm that the phase crossover frequency of $P_{K}$ is improved to about $20.9(\mathrm{rad} / \mathrm{s})$, therefore the specification 1 is fully achieved. 
In Fig. 7, phase/gain constraint (19), (20) and (21) are shown as hatched areas. Note that the phase constraint (19) is meaningful in the specified bandwidth (17). Therefore, the finite bandwidth phase shaping technique gives us more reasonable phase shaping than the technique based on $\gamma$-positive realness.

\section{CONCLUSION}

This paper discussed the vehicle control augmentation strategy from the viewpoint of the phase bandwidth of the vehicle dynamics. Furthermore, the finite bandwidth phase shaping technique is proposed and applied to the phase crossover bandwidth shaping for the CAS (control augmentation system) design. From the reconsideration of Neal and Smith's handling quality investigation, it is observed that phase crossover bandwidth plays important role to improve the handling quality. However, the phase shaping design in the authors' preliminary work results in the phase shaping in all frequency range, though the plant phase property in the very high frequency is noncrucial for control performance. Therefore, in this paper, the authors proposed another tractable phase shaping technique in the finite bandwidth via dissipative control theory. The design problem is reduced to a LMI problem and the design example of the aircraft pitch pointing CAS is shown to demonstrate the effectiveness of the proposed technique.

\section{REFERENCES}

Bihrle Jr., W. (1966). A handling qualities theory for precise flight path control. AFFDL-TR65-198.

Davidson, J. B. and D. K. Schmidt (1992). Modified optimal control pilot model for computeraided design and analysis. TM-4384.

Doman, D. B. and M. R. Anderson (2000). A fixed-order optimal control model of human operator response. Automatica 36, 409-418.

Grigoriadis, K. M. and F. Wu (1997). Integrated $h_{\infty}$ plant/controller design via linear matrix inequalities. In: Proc. of 36th IEEE CDC. pp. 789-790.

Iwasaki, T. (1999). Integrated system design by separation. In: Proc. of IEEE CCA'99. pp. $97-102$.

Iwasaki, T., G. Meinsma and M. Fu (2000). Generalized $S$-procedure and finite frequency KYP lemma. Math. Prob. Eng. 6, 305-320.

Kleinman, D., S. Baron and W. Levison (1970). An optimal control model of human response. part 1: Theory and validation. Automatica 6, 357-369.
Military Specification (1990). Department of defense interface standard, flying qualities of piloted aircraft. MIL-STD-1797A. Notice of change, 28 June 1995.

Neal, T. P. and R. E. Smith (1971). A flying qualities criterion for the design of fighter flightcontrol systems. AIAA J. Aircraft 8, 803-809.

Onoda, J. and R. Haftka (1987). An approach to structure/control simultaneous optimization for large flexible spacecraft. AIAA Journal 25, 1133-1138.

Sakamoto, N. and M. Suzuki (1996). $\gamma$-passive system and its phase property and synthesis. IEEE Trans. Auto. Contr. 41, 859-865.

Satoh, A., M. Takei and K. Sugimoto (2004). Vehicle control augmentation based on an integrated design of mechanical control systems. In: Proc. of MTNS2004.

Schmidt, D. K. (1979). Optimal flight control synthesis via pilot modeling. J. Guid. Contr. 2, 78-1286.

\section{ACKNOWLEDGMENT}

This work was supported in part by Tateisi Science and Technology Foundation. 